\title{
Simulation and interpretation of inter-well tracer tests
}

\author{
Olaf Husebya , Jan Sagen, Sissel Viig and Øyvind Dugstad \\ Institutt for energiteknikk, Kjeller Norway
}

\begin{abstract}
In inter-well tracer tests (IWTT), chemical compounds or radioactive isotopes are used to label injection water and gas to establish well connections and fluid patterns in petroleum reservoirs. Tracer simulation is an invaluable tool to ease the interpretation of IWTT results and is also required for assisted history matching application of tracer data. In this paper we present a new simulation technique to analyse and interpret tracer results. Laboratory results are used to establish and test formulations of the tracer conservation equations, and the technique is used to provide simulated tracer responses that are compared with observed tracer data from an extensive tracer program. The implemented tracer simulation methodology use a fast post-processing of previously simulated reservoir simulation runs. This provides a fast, flexible and powerful method for analysing gas tracer behaviour in reservoirs. We show that simulation time for tracers can be reduced by factor 100 compared to solving the tracer flow equations simultaneously with the reservoir fluid flow equations. The post-processing technique, combined with a flexible built-in local tracer-grid refinement is exploited to reduce numerical smearing, particularly severe for narrow tracer pulses.
\end{abstract}

\section{INTRODUCTION}

Tracer testing in petroleum reservoirs is a mature technology, where fluid movement is tracked by tagging injection fluids by inert chemical or radioactive compounds (for a review see [1]). One application of tracers is the inter-well tracer test (IWTT), which is used to obtain information on well-to-well communication, heterogeneity and fluid dynamics [2]. One of the appealing features of IWTT is that at the first tracer breakthrough in a producer, immediate and unambiguous information on the injector - producer communication is given. In cases with several sources of injection fluids, tracers yield unique information on the origin of fluids. During IWTT injected fluids are labeled using unique radioactive or chemical tracer compounds, which are then subsequently used to trace the fluids as they move through the reservoir. Many compounds may a priori be candidates for tracer application. However, compounds are subject to harsh temperature and pressure conditions, biodegradation, scaling reactions, ion exchange, adsorption to the solid phase etc. Modeling of these effects would require a detailed description of the chemical and physical conditions in the reservoir, conditions that are generally not known. Rather than attempting to model such effects, it is customary to use tracers that behave in a predictable manner. This excludes many potential tracers, and few tracers are actually available for use in oil reservoirs. Common water tracers are tritiated water (HTO) and fluorinated benzoic acids, common gas tracers are tritiated methane or perfluorinated carbons (PFC) [3]. In tritiated tracer molecules, a stable hydrogen atom is replaced by its radioactive isotope (tritium). In the fluorinated tracers carbon atoms are replaced by fluor atoms. The latter group of tracers are commonly referred to as chemical tracers, in contrast to the former radioactive tracers. Due to regulatory issues radioactive tracers are sometimes avoided, although environmental impact of either group of tracers is small.

To use tracer data for reservoir simulation model improvement it is important to model the tracer transport

a e-mail: olaf .huseby@ife.no correctly. This requires an adequate formulation of the tracer conservation equations, as well as satisfactory numerical methods. For tracers, numerical accuracy is particularly important, because tracer injections are narrow pulses, i.e. with small time and length scales compared to those used in a typical reservoir simulation set-up. Significant refinement of the reservoir grid used for solving the Darcy flow and pressure in a reservoir simulation is not feasible in real-life cases, as the execution times of unrefined cases are usually in the order of one day and refinement of the reservoir grid increases the execution times significantly. In the following we will show how the solution of the tracer-problem can be achieved at a satisfactory accuracy without too severe consequences for the execution times.

\section{FORMULATION AND NUMERICAL SOLUTION OF TRACER TRANSPORT EQUATIONS}

In IWTTs tracers are injected in small quantities and are present in the reservoirs at minuscule concentrations. Tracers are subject to several physical and chemical effects, and the most important ones are partitioning between liquid phases, adsorption to the solid phase, diffusion and dispersion and radioactive decay.

Radioactive decay can easily be accounted for by correcting measured concentrations by decayed quantities, and are thus not considered here. Adsorption of the solutes onto the rock surface may also be important, especially in the presence of clay minerals. Adsorption complicates the interpretation of tracer data and in general one avoid tracers that interact significantly with the solid matrix in the reservoirs. Molecular diffusion may be important, especially when fluid velocities are close to zero. Good estimates for molecular diffusion coefficients $D_{m, w}$ and $D_{m, o}$ of tracers can be obtained from experimental correlations such as the Wilke-Chang and Hayduk-Mindas expressions [4]. Dispersion is larger than diffusion when the dimensionless Péclet number is non-zero. It is a tensor quantity with different components along and transversal 
to the flow direction. The dispersion coefficients are functions of the Péclet number, and these functions are sometimes assumed to be linear in the Péclet number. If this is the case, the so-called dispersivity can be defined and used as an alternative formulation to incorporate dispersion.

Partitioning between liquid phases is important for some tracer applications. These include single-well and interwell partitioning tracer tests, where oil/water partitioning tracers are used to assess oil saturations. In addition, gas tracers in reservoirs always partition between the oil and gas phases. For water tracer tests, partitioning can be neglected, as reliable non-partitioning water tracers (e.g. HTO and fluorinated benzoic acids) are available. Partitioning coefficients can be determined in the laboratory. A partitioning tracer is by definition a tracer which exists in two or more phases simultaneously. The concentration distribution within the phases is governed by partitioning coefficients specific for each tracer and the composition of the fluids in the phase. In general, partitioning coefficients are temperature and pressure dependent, but this can usually be neglected in most cases. This is a valid simplification if temperature and pressure gradients are not too severe.

For a water tracer $p$ the partitioning coefficient (K-value) between oil and water is defined by:

$$
K^{p}=\frac{C_{o}^{p}}{C_{w}^{p}},
$$

where $C_{o}^{p}$ and $C_{w}^{p}$ are the concentrations of the tracer $p$ in the oil and water phase, respectively. For a gas tracer $r$ the partitioning coefficient between oil and gas is defined in a similar manner:

$$
K^{r}=\frac{C_{o}^{r}}{C_{g}^{r}} .
$$

To cover all the cases of a tracer partitioning between three phases, it is convenient to apply an absolute $\mathrm{K}$-value in the tracer conservation equation. The $\mathrm{K}$-value for the primary phase is set to 1 , and the other $\mathrm{K}$-values are similar to the relative K-values defined in Eqs. (1) and (2).

The conservation equation for an arbitrary tracer component may now be written

$$
\begin{aligned}
& \frac{\partial}{\partial t}\left(\sum_{i=o, g, w} \phi K_{i} S_{i} C\right)+\nabla \cdot\left(\sum_{i=o, g, w} \boldsymbol{v}_{i} K_{i} C\right) \\
& -\nabla \cdot\left(\sum_{i=o, g, w} \phi S_{i}\left(\frac{D_{m, i}}{\tau} \boldsymbol{I}+\boldsymbol{D}_{i}^{*}\right) \cdot \nabla\left(K_{i} C\right)\right)=0
\end{aligned}
$$

where $C$ is concentration of the tracer, $\phi$ is porosity, $S_{i}$ is phase saturation $\left(S_{w}, S_{o}\right.$ or $\left.S_{g}\right), \boldsymbol{v}_{i}$ is the velocity of phase $\mathrm{i}, \tau$ is tortuosity, $D_{m, i}$ is the solute molecular diffusion coefficient in phase $i$ and $\boldsymbol{I}$ is the identity tensor. The dispersion tensor $\boldsymbol{D}_{i}^{*}$ can be written as

$$
\boldsymbol{D}_{i}^{*}=D_{m, i}\left\{D_{i \|}^{*} \boldsymbol{e}_{\|} \boldsymbol{e}_{\|}+D_{i \perp}^{*}\left(\boldsymbol{I}-\boldsymbol{e}_{\|} \boldsymbol{e}_{\|}\right)\right\},
$$

where $\boldsymbol{e}_{\|}$is a unit vector along the flow velocity, and $D_{m, i}$ is the solute molecular diffusion coefficient. The coefficients $D_{i \|}^{*}$ and $D_{i \perp}^{*}$ depend on the flow velocity of phase $i$ and a typical length scale (e.g. the bead-size in spherical packings) $d$ through the Péclet number

$$
\mathrm{Pe}_{i}=\frac{v_{i} d}{D_{m, i}}
$$

and can be approximated by power laws [5]

$$
D_{i \|}^{*}=\beta_{\|} \mathrm{Pe}^{\alpha_{\|}}, \quad D_{i \perp}^{*}=\beta_{\perp} \mathrm{Pe}^{\alpha_{\perp}} .
$$

The exact expression for the phase velocities $\boldsymbol{v}_{i}$ is simulator-dependent, for instance by assuming Darcy flow with gravity,

$$
\boldsymbol{v}_{i}=-\frac{\boldsymbol{k} k_{r}^{i}}{\mu_{i}} \cdot\left(\nabla P_{i}-\rho_{i} \boldsymbol{g}\right),
$$

where $\boldsymbol{k}$ is the absolute permeability tensor, $\mu_{i}$ the viscosity, $k_{r}^{i}$ the relative permeability, $\nabla P_{i}$ the pressure gradient in phase $i, \rho_{i}$ the density of phase $i$ and $\boldsymbol{g}$ the gravitational acceleration.

For a non-partitioning water tracer, $K_{w}=1$ and $K_{o}=$ $K_{g}=0$, and Eq. (4) reduces to

$$
\begin{aligned}
& \frac{\partial}{\partial t}\left(\phi S_{w} C\right)+\nabla \cdot\left(\boldsymbol{v}_{w} C\right) \\
& -\nabla \cdot\left(\phi S_{w}\left(\frac{D_{m, w}}{\tau} \boldsymbol{I}+\boldsymbol{D}_{w}^{*}\right) \cdot \nabla\right)=0 .
\end{aligned}
$$

In addition to the phase velocities $\boldsymbol{v}_{i}$ the tracer transport described by Eq. (4) depends on saturations $S_{i}$. However, due to the minuscule amounts of tracers injected in the reservoir, the tracers do not affect the fluid transport in the reservoir. This is an important observation that can be used to decouple the tracer simulation from the reservoir simulation.

Since tracers do not influence the fluid flow in the reservoir, simulation of tracer transport may be performed in a separate module decoupled from the reservoir simulation itself. This was exploited by Sagen and co-workers [6], who solved for tracer transport using fluid flow solutions obtained from an ordinary reservoir simulation. In [6] the flow field was retrieved from the host reservoir simulator at each time-step, and then used to find tracer flow simultaneously with solving the fluid flow. This modular method allows coupling to any black oil or compositional reservoir simulators, if the necessary information can be obtained from the host reservoir simulator. It also opens for a separate time and space discretization of the tracer equation, which was used in [6] to obtain accurate solutions.

Numerical smearing is a general problem that occur when transport equations are solved numerically. Smearing of the numerical solution occurs both for saturation fronts and tracer fronts, but are particularly severe when solving the transport equations for tracer pulse injections. Various kinds of spatial and temporal weighting as well as flux limiter schemes have been developed to counter numerical smearing. Application of spatial weighting is relatively straight forward, whereas temporal weighting may cause instability effects unless good time step control 
is implemented that ensures that the Courant-FriedrichsLewy (CFL) condition is satisfied. Even if time step control is implemented and CFL-conditions satisfied, this may restrict the tracer simulator to unpractical small time-steps. For this reason an implicit solution was chosen for the implementation. Unfortunately, the implicit formulation is particularly prone to numerical smearing, which may lead to severe effects in the tracer solution.

To improve on this situation, one can use successively finer time-steps and a separately refined tracergrid. Since the tracer transport is separated from the flow problem solved by the host reservoir simulator, a smaller time-step and refined grid can be used without affecting the reservoir simulator.

\section{DEMONSTRATION IN A LABORATORY CASE}

The tracer simulation methodology was tested using laboratory data. In a laboratory experiment, water was pumped into a water-filled column packed with sand (Sibelco), at a constant rate of $1.374 \mathrm{ml} / \mathrm{h}$. The column is oriented along gravity, and water pumped from the bottom face of the column. The porosity of the column is $44 \%$ and the permeability is about 3 Darcy. The length of the column is $L_{z}=25 \mathrm{~cm}$ and the diameter is $D=2.1 \mathrm{~cm}$. Tritiated water (HTO) was used as an ideal tracer and was injected at the lower interface of the column. During the experiment the column was kept at constant temperature $\mathrm{T}=323.15 \mathrm{~K}$. The geometry of the experiment is illustrated in Figure 1.

Water samples were collected at the outlet and concentrations were measured. During transport through the column the HTO tracer is subject to convection with the water and dispersion due to molecular diffusion and irregularities in the sand-pack. The combined effect of diffusion and dispersion of the HTO-tracer is given by the diffusion coefficient and Eq. (4). In sandstone $1 / \tau \sim 0.7$ [7] and we get a diffusion-dispersion term equal to $D_{m} \times$ $\left(0.7+\beta \mathrm{Pe}^{\alpha}\right)$. Here $\mathrm{Pe}=v_{i} d_{s} / D_{m}$ and $v_{i}$ is interstitial velocity, $d_{s}$ are grain sizes and $D_{m}$ is molecular diffusion. Molecular diffusion for HTO is $3.9 \times 10^{-9} \mathrm{~m}^{2} / \mathrm{s}$ at T $=$ $323.15 \mathrm{~K}$ [8], the grain size is about 200 microns and the interstitial velocity is about $2.5044 \times 10^{-6} \mathrm{~m} / \mathrm{s}$. This yields a Péclet number of about 0.1 . For such small Péclet number the diffusion-dispersion term is thus dominated by $D_{m} / \tau=D_{m} \times 0.7 \sim 2.7 \times 10^{-9} \mathrm{~m}^{2} / \mathrm{s}$.

The tracer transport in this experiment is well described by a 1-D convection dispersion equation (obtained from Eq. (4)).

$$
\frac{\partial C}{\partial t}+v \frac{\partial C}{\partial x}-D_{x}^{*} \frac{\partial^{2} C}{\partial x^{2}}=0
$$

with the initial and boundary conditions

$$
\begin{aligned}
& C(x=0, t)=\frac{M}{Q} \delta(t) \\
& C(x, t=0)=0 \\
& \lim _{x \leftarrow \infty} C(x, t)=0 .
\end{aligned}
$$

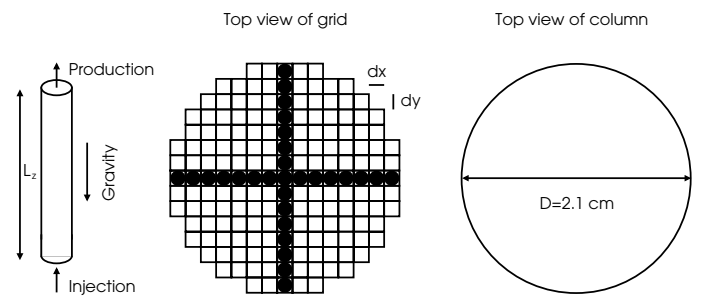

Figure 1. Geometry of tracer experiment used to demonstrate the simulation methodology, and grid used to simulate the experiment.

This has the solution [9]

$$
C(x, t)=\frac{M}{Q} \frac{x}{\sqrt{4 \pi D_{x} t^{3}}} \exp \left[-\frac{(x-v t)^{2}}{4 D_{x} t}\right]
$$

Equation (11) yields the concentration as function of time at the producer

$$
C_{p}(t)=\frac{M}{Q} \frac{L}{\sqrt{4 \pi D_{x} t^{3}}} \exp \left[-\frac{(L-v t)^{2}}{4 D_{x} t}\right] .
$$

The analytical result was obtained from Eq. (12) with $L=L_{z}=0.25 m, D_{x}=2.7 \times 10^{-9} \mathrm{~m}^{2} / \mathrm{s}$, and $v=v_{i}=$ $2.5044 \times 10^{-6} \mathrm{~m} / \mathrm{s}$.

Numerical solutions were obtained using the same procedure as for Example 1 above. The flow equations were solved by the host simulator using 60 gridblocks in the $\mathrm{z}$-direction $\left(\delta_{z}=0.25 / 60 \mathrm{~m}\right)$ and the grid geometry illustrated in Figure 1 in $\mathrm{x}$ and $\mathrm{y}$ directions, with gridblock sizes $\Delta_{x}=\Delta_{y}=0.0013989 \mathrm{~m}$. The tracer transport equation was then solved using a separate tracer grid and separate time-step with successive refinement of tracer gridblocks and time-steps. The grid refinement was done in the z-direction using tracer gridblock sizes of $\delta_{x}=\Delta_{x} / n_{x}$, where $n_{z}=1,5,9$ and 15 , excluding the well blocks. The refined tracer time-steps used were $d_{t}=0.1$, $1 \times 10^{-2}, 1 \times 10^{-3}$ and $1 \times 10^{-4}$ days.

The measured concentrations are summarized in Figure 2 and compared with simulation results and the analytical solution. If we focus first on the analytical solution we see that it compares very well with the experimental data, which shows (as expected) that the assumption of a 1-D solution is sound.

Result of the refinement in z-direction for a fixed time-step $d_{t}=1 \times 10^{-4}$ days, is displayed in Figure $2 \mathrm{a}$, and result of the time refinements for a fixed grid refinement $n_{z}=9$ is displayed in Figure $2 \mathrm{~b}$, and the results obtained without refinement in space and time are given for comparison. From Figure 2 it is clear that decreasing the tracer grid size and tracer time-step improves the numerical solution. The numerical solutions approach the analytical solution and the measured results in a similar manner as for the large scale case given in Example 1.

\section{DEMONSTRATION IN A FIELD CASE}

Recently, Rwechungura and co-workers [10] published data from the E-segment of Norne Field on the Norwegian 

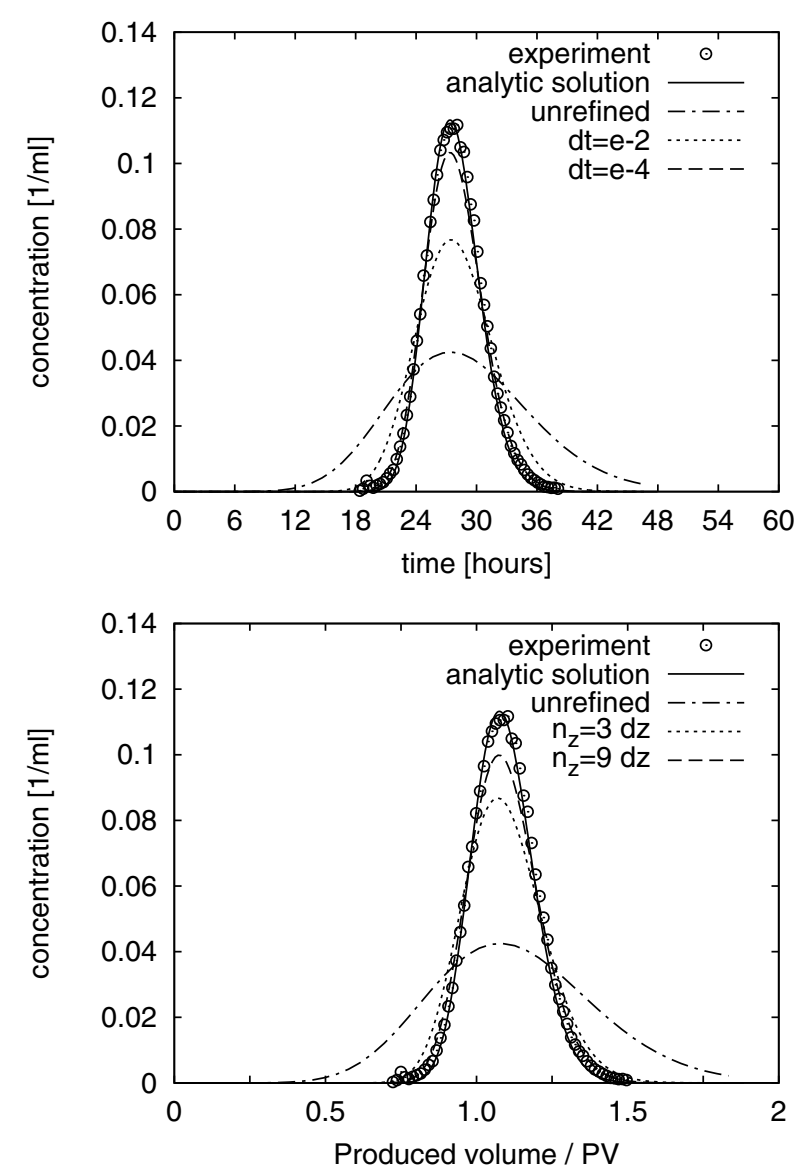

Figure 2. Tracer concentrations from the laboratory experiment, compared to the analytic solution Eq. (12), and numerical solutions obtained with several refinements in the time-step and tracergrid.

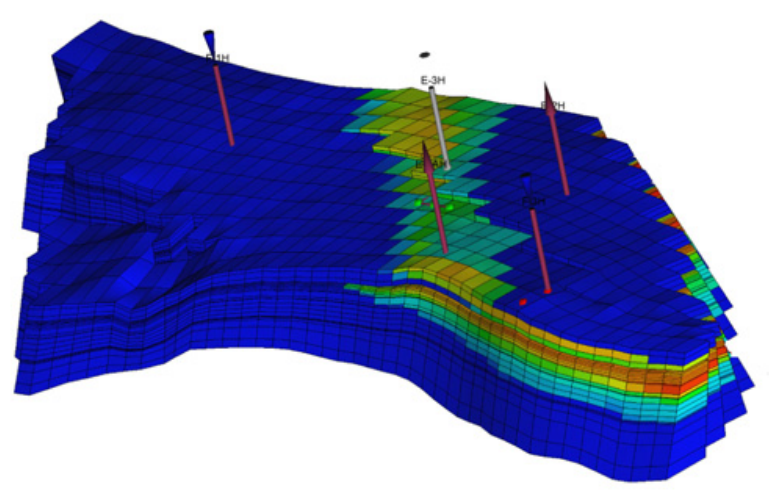

Figure 3. Illustration of the E-segment of the Norne benchmark case provided by [10]. The original grid is retained in the Esegment, and coarsened gridblocks used for the rest of the field.

continental shelf. This is a real-field benchmark case made available to the petroleum research community as download from the Norne benchmark website. The case was derived from a full field model (see Fig. 3a) in [10] and is given as a set of input files to the commercially available Eclipse simulator. Outside the E-segment of the model, gridblocks are coarsened whereas in the E-segment the gridblock sizes of the original case was retained (see Fig. 3b). The original case has 44431 active cells and the

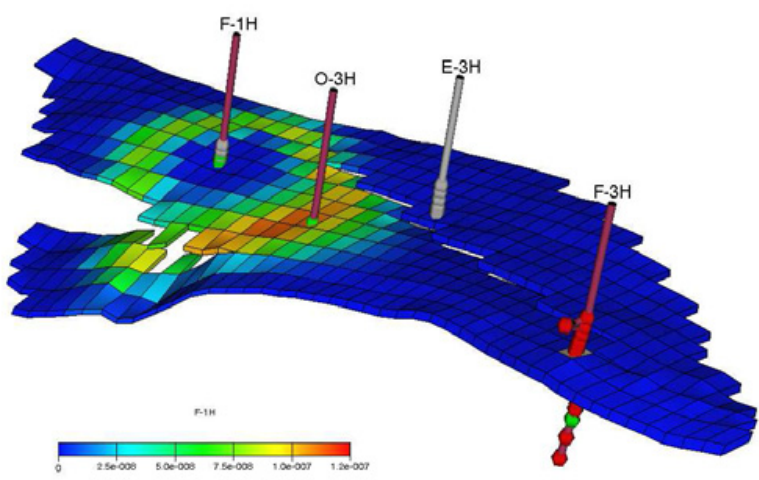

Figure 4. Concentration of tracer $\mathrm{TF}-1 \mathrm{H}$ in layer 10 of the reservoir grid in the E-segment of the Norne benchmark case. The tracer is injected in F-1H on September 3, 1999 and the figure shows concentrations in July 2003, corresponding to the time of breakthrough in observation well $\mathrm{O}-3 \mathrm{H}$.

benchmark case has 9771 active cells. For further details on the Norne benchmark case we refer to [10].

In the Norne benchmark case obtained from the website, production and injection data from 6th of November 1997 until 31st of December 2004 is provided. Measured tracer data are not given for the benchmark case. For the purpose of testing the tracer simulation methodology a tracer injection in injector $\mathrm{F}-1 \mathrm{H}$ was therefore assumed. The tracer is denoted TF- $1 \mathrm{H}$ and is a non-partitioning water tracer, assumed to be subject to a constant and isotropic dispersion $\boldsymbol{D}_{w}^{*}=\boldsymbol{I} \cdot 1 \cdot 10^{-5} \mathrm{~m}^{2} / \mathrm{s}$, similar to the one used in the 1-D reservoir scale example above. The injector $\mathrm{F}-1 \mathrm{H}$ where the tracer is injected spans several layers and tracer movement therefore occur in many layers. Consequently the tracer production curve in the nearest producer E-3H is rather complex. To simplify the interpretation of the simulation tests an observation well $(\mathrm{O}-3 \mathrm{H})$ was therefore introduced and located to a single gridblock. The observation well produces a small, constant amount of water, where the $\mathrm{F}-1 \mathrm{H}$ tracer is sampled.

In order to study the tracer curves after 2004, the field's injectors and producers were assumed to continue with constant rates for two years after December, 2004. Results after 2004 are thus not reflecting the fields real injection and production schedule. Prior to the tracer simulations, the reservoir flow equations were solved for the Norne benchmark case using the Eclipse simulator. The solutions were saved and used for the subsequent tracer simulations. The tracer simulations were then performed by the same post-processing procedure as described previously. A base-case tracer simulation was done using the same time-step and the same grid as that used to solve the reservoir flow simulation. Figure 4 gives a plot of the tracer concentrations from the base-case tracer simulation. Concentrations are given in layer 10 of the grid in June, 2001, corresponding roughly to the time when concentration peaks in the observation well $\mathrm{O}-1 \mathrm{H}$. The injectors $\mathrm{F}-1 \mathrm{H}$ and $\mathrm{F}-3 \mathrm{H}$ and the producer $\mathrm{E}-3 \mathrm{H}$ are also visible in the figure.

Once the base-case tracer simulation was obtained, using a tracer grid equal to the reservoir grid, the tracer simulation was repeated, but with refinement of the tracer 


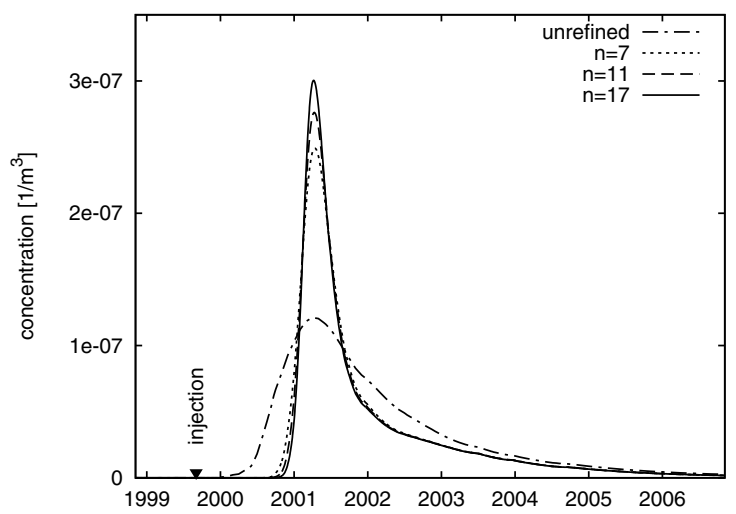

Figure 5. Test of grid refinement in the Norne field case, for a fixed small time-step $d_{t}=0.1$ days. Tracer results obtained without refinement in space and time are given for comparison.

grid as well as the tracer time-step. Tracergrid refinement was used in 3576 of the 9771 original reservoir blocks in the region between injector $\mathrm{F}-1 \mathrm{H}$ and observation well $\mathrm{O}-3 \mathrm{H}$. The grid refinement was done in the $\mathrm{x}$ and $\mathrm{y}$ directions using tracer gridblock sizes of $\delta_{x}=\Delta_{x} / n$ and $\delta_{y}=$ $\Delta_{y} / n$, where $n=1,3,5$ and 7. The number of blocks in the tracergrid are given as $N_{b}=9771+3576\left(n^{2}-\right.$ 1). Result of the tracergrid refinements is displayed in Figure 5. The results obtained without grid and time refinement are given for comparison. From Figure 5 it is clear that decreasing the tracer grid size improves the numerical solution significantly.

\section{CONCLUDING REMARKS}

A new tracer simulation technique has been introduced, where the most important characteristic is that the tracer simulation is performed in a post-processing manner, based on pre-calculated solutions of the fluid flow obtained from reservoir simulation. The technique can be used with any reservoir simulator that can store fluid flow results in an accessible format. The technique has been tested using pre-calculated reservoir simulation results from the Eclipse simulator for one laboratory case, two synthetic reservoir cases and the Norne benchmark reservoir case.

The Norne case illustrated that tracer simulation using the new simulation technique is very fast (a few \%) compared to tracer simulation performed simultaneously with a reservoir simulation. Because additional tracer simulations do not require re-running the reservoir simulator, lessons-learned from one tracer simulation can be efficiently used in subsequent simulations. This is valuable in planning of tracer injections, and also in evaluations of tracer results.

Numerical dispersion is a general problem and particularly important for tracers. By using the new tracer simulation technique it was demonstrated that successive refinement, based on a single reservoir simulation can be use to establish the correct tracer solution. In one of the synthetic reservoir case numerical solutions were refined until they represented well the analytical solution to the tracer transport equation. In an experimental case grid and time refinement provided numerical solutions that compares very well with the experimental results. Finally, using the real Norne reservoir case, it was demonstrated that successive refinement can provide reliable numerical solutions. Separate tracer grid refinement was used in all cases, and was shown to be particularly useful in the field case, where significant refinement of the reservoir grid is not feasible. In the field case, testing of grid refinement in a large number of reservoir grid-blocks and a smaller number of grid-blocks show that identification of the most important zones to refine is feasible. This is important, because even the fast, separate tracer-grid refinement becomes costly in terms of CPU times for significant refinements in time-step and space. The implemented method uses an implicit up-wind method for the tracerflow. More accurate schemes, less prone to numerical smearing, while retaining the stability are available, and is the subject of future implementation.

Financial contribution from the Research Council of Norway, Statoil and Total, and licenses to run the Eclipse reservoir simulator provided by Schlumberger, is gratefully acknowledged. We also acknowledge Statoil (operator of the Norne field) and its license partners ENI and Petoro for the release of the Norne data and the Center for Integrated Operations at NTNU for providing the Norne Case.

\section{References}

[1] Zemel B., Tracers in the Oil Field (Elsevier, New York, 1994)

[2] Dugstad Ø., "Well-to-Well Tracer Tests". Chapter 6 (pp. 651-683), Petroleum Engineering Handbook, Vol. 5 - Reservoir Engineering and Petrophysics by Edward D. Holstein (Ed.) SPE, Richardson, Texas, 2007

[3] Dugstad Ø., Bjørnstad T., and Hundere I.A., "Measurements and Application of Partition Coefficients of Compounds Suitable for Tracing Gas Injected into Oil Reservoirs". Revue de l'Institut Francais du Pétrole 47 (2), 205-215 (1992)

[4] Perry R.H., Green D.W., and Maloney J.O., Perry's chemical engineers' handbook 7th ed. McGraw-Hill, New York, 1997

[5] Adler P.M.: Porous media : geometry and transports. (Butterworth/Heinemann, Stoneham, MA, 1992)

[6] Sagen J., Cvetkovic B., Brendesdal E., Halvorsen G., You Y.L., and Bjornstad T., "Reservoir ChemicalThermal Simulation with Tracers". SPE paper 36921, SPE European Petroleum Conference, Milan, Italy, 22-24 October, 1996

[7] Dullien F.A.L.; "Porous media: fluid transport and pore structure" Academic Press, London, 1992

[8] Mills R.: "Self-Diffusion in normal and Heavy-Water in the Range 1-45 Degrees". Journal of Physical Chemistry 77 (5), 685-688 (1973)

[9] Lenda A., and Zuber A., "Tracer dispersion in groundwater experiments." In: Isotope Hydrology (Proc. IAEA Symp.), IAEA, Vienna, pp 619-641, 1970

[10] Rwechungura R., Suwartadi E., Dadashpour M., Kleppe J., and Foss B., "The Norne Field Case - A Unique Comparative Case Study" SPE paper 127538. SPE Intelligent Energy Conf., 23-25 March Utrecht, The Netherlands, 2010 